ẼESTI NSV TEADUSTTE AḰADEEMIA TOIMETISED, 26. KOIDÉ FOOSIKA * MATEMAATIKA. 1977, NR. 3

ИЗВЕСТИЯ АКАДЕМИИ НАУК ЭСТОНСКОЙ ССР. ТОМ 26 ФИЗИКА * МАТЕМАТИКА, 1977, № 3

\title{
ТЕОРИЯ ПЕРЕХОДНЫХ СПЕКТРОВ РЕЗОНАНСНОГО ВТОРИЧНОГО СВЕЧЕНИЯ ПРИМЕСНЫХ ЦЕНТРОВ КРИСТАЛЛОВ
}

\section{1. Введение}

Исследование процессов релаксации и, в частности колебательной релаксации, является одной из актуальных задач физики кристаллов и молекул настоящего времени. Создание лазерных систем с длительностью импульсов в пикосекундном диапазоне позволило поставить ряд экспериментов по непосредственному наблюдению временного хода таких процессов в интервале $t \sim 10^{-12}$ сек (см., напр., $\left[{ }^{1-3}\right]$ и обзоры $[4,5])$. Появились также сообщения о создании в ближайшем будущем лазерных систем, дающих импульсы длительностью $10^{-13}$ сек (см., напр., $\left.\left[{ }^{6}\right]\right)$, т. е. короче периодов колебаний многих кристаллов и молекул. Это делает весьма актуальным развитие теории временного хода как самих процессов колебательной релаксации, так и тех физических явлений, в которых она может наблюдаться. Одним из таких явлений, в которых колебательная релаксация проявляется самым непосредственным образом, является резонансное вторичное свечение (РВС) примесных центров в кристаллах и центров люминесценции в растворах.

Ранее $\left[{ }^{7-10}\right]$ (см. также обзоры $\left[{ }^{11,12}\right]$ ) теория РВC примесных центров кристаллов была развита в стационарных условиях возбуждения. Было показано, что основой теории, совместно описывающей все компоненты РВС, может служить формула второго порядка по взаимодействию света и вещества для сечения резонансного рассеяния (формула (33а) данной работы). В тех случаях, когда колебательная релаксация в возбужденном электронном состоянии центра происходит гораздо быстрее затухания этого состояния (а именно таким свойством обладают известные центры), основная по интенсивности часть РВС, описываемого этой формулой, представляет собой обычную люминесценцию (ОЛ). (Это было впервые доказано в $\left[{ }^{7}\right]$ и в последующем подтверждено в $\left[{ }^{13-16}\right]$ и др.) Кроме того, отмеченная формула содержит более слабые резонансные релеевское и комбинационное рассеяния, горячую люминесценцию (ГЛ), а также интерференционные поправки к ним $\left[{ }^{7-9}\right]$. ОЛ испускается после колебательной релаксации в возбужденном электронном состоянии, остальные компоненты РВС испускаются до или в процессе колебательной релаксации и имеют спектры, существенно отличные от спектра ОЛ *. Поэтому спектр РВС

* В $\left[{ }^{17}\right]$ (см. также $\left.\left[{ }^{9}\right]\right)$ был предложен следующий критерий классификации резонансного комбинационного рассеяния (РКР) и ГЛ: РКР испускается до фазовой (и энергетической) колебательной релаксации в возбужденном электронном состоянии, а ГЛ - после фазовой, но в процессе энергетической колебательной релаксации. Поскольку фазовая и энергетическая релаксации происходят совместно (хотя и имеют часто существенно разную скорость), разделение РКР и ГЛ возможно лишь приближенно. Однако во мнопих случаях указанная неоднозначность разделения ГЛ и РКР практически не существенна. 
действительно должен существенно зависеть от времени, а эта зависимость должна содержать детальную информацию о процессе колебательной релаксации в возбужденном электронном состоянии. В связи с этим представляет интерес развить теорию временной зависимости спектра РВС примесных центров кристаллов, позволяющую выяснить, какая конкретно информация может быть получена из такого рода спектров.

Следует отметить, что развитие теории временной зависимости РВС важно и для проблемы классификации компонентов РВС, ставшей в последнее время особенно актуальной в связи с широким развертыванием исследований по РКР. Использование на эксперименте для классификации РВС временных характеристик не только полезно, но и в ряде случаев необходимо. Как показано в $\left[{ }^{18}\right]$, к таким системам относятся растворы, а также все другие системы со значительным неоднородным уширением, классификация РВС по спектральным признакам в которых практически невозможна. Напомним также, что именно временные характеристики вторичного свечения были положены С. И. Вавиловым в основу классификации вторичного свечения $\left[{ }^{19}\right]$.

Целью данной работы является детальное развитие теории, позволяюей описать зависимость от времени характеристик мгновенного (переходного) спектра РВС примесных центров, испускаемого в процессе колебательной релаксации в возбужденном электронном состоянии. Нами получена общая формула, определяющая переходный спектр РВС. Исходя из этой формулы в рамках определенной модели центра найдено асимптотически точное выражение для сглаженного переходного спектра. Выполнены также конкретные расчеты спектров ГЛ (и ОЛ), испускаемых на различных этапах релаксации, наглядно иллюстрирующие, каким образом в переходных спектрах РВС проявляются процессы колебательной релаксации.

\section{2. Переходный спектр РВС}

Наша задача - исследовать зависимость от времени сечения резонансного рассеяния света центром люминесценции кристалла (раствора). Рассматриваемый процесс является двухфотонным и, как отмечалось выше, описывается формулой второго порядка по взаимодействию света и вещества. Обычный вывод этой формулы (см. $\left.\left[{ }^{20}\right]\right)$ основывается на решении уравнения Шредингера для взаимодействующих электромагнитного поля и вещества при начальных условиях, заданных в момент времени $t=0$. Однако это состояние, как правило, не известно. Действительно, реальное возбуждение системы осуществляется волновыми пакетами электромагнитного поля, имеющими конечные размеры в пространстве и во времени. Поэтому фактически известным можно считать состояние поля и вещества лишь в момент времени $t_{0}=-\infty$, когда волновые пакеты поля и вещества не перекрываются (в пространстве) и не взаимодействуют. В любой же конечный момент времени пакеты перекрываются и, следовательно, взаимодействуют. Лишь в случае специальной формы пакетов взаимодействие может начинаться в конечный момент времени (который и можно выбрать за начало отсчета времени). В стационарных условиях возбуждения и при спонтанном механизме испускания вторичного фотона форма пакета первичных фотонов не играет роли. Поэтому полученная в $\left[{ }^{20}\right]$ формула является общей. Однако при анализе временных характеристик РВС форма возбуждающего пакета важна, вследствие чего формулу $\left[{ }^{20}\right]$ для вероятности двухфотонного процесса при конечных временах в общем слу- 
чае использовать нельзя. Ее следует видоизменить таким образом, чтобы в качестве исходного фигурировало состояние поля и вещества в момент времени $t_{0}=-\infty$. Это может быть сделано следующим образом.

Если система, описываемая гамильтонианом $H=H_{(0)}+V$, в начальный момент времени $t_{0}$ находится в состоянии

$$
\left|\psi\left(t_{0}\right)\right\rangle=\sum_{j^{\prime}} d_{j^{\prime}}\left|j^{\prime}\right\rangle \mathrm{e}^{-i E_{\gamma^{\prime}} t_{0},} \quad \bar{\pi}=1,
$$

гдс $\left|j^{\prime}\right\rangle$ - собственные состояния, а $E_{j^{\prime}}$ - собственные значения нулевого гамильтониана $H_{(0)}$, то амплитуда вероятности $c_{j}(t)$ найти систему в момент времени $t \geqslant t_{0}$ в состоянии $|j\rangle$ согласно уравнению Шредингера будет равна

$$
c_{j}(t)=\left\langle j\left|\mathrm{e}^{-i\left(t-t_{0}\right) H}\right| \psi\left(t_{0}\right)\right\rangle=\sum_{j^{\prime}}\left\langle j\left|\mathrm{e}^{-i\left(t-t_{0}\right) H}\right| j^{\prime}\right\rangle \mathrm{e}^{-i E_{j^{\prime}} t_{0}} d_{j^{\prime}} .
$$

Учтем, что при $x \geqslant 0$

$$
\mathrm{e}^{-i x H}=\mathrm{e}^{-i x H_{(0)} T} \exp \left\{-i \int_{0}^{x} V(s) d s\right\}
$$

где $V(s)=\exp \left(i s H_{(0)}\right) V \exp \left(-i s H_{(0)}\right), T-$ знак хронологического упорядочения операторов справа налево в порядке возрастания $s$. Тогда выражение (2) можно переписать в виде

$$
\begin{aligned}
c_{j}(t)= & \sum_{n=1}^{\infty}(-i)^{n} \int_{t_{0}}^{t} d t_{1} \int_{t_{0}}^{t_{1}} d t_{2} \ldots \int_{t_{0}}^{t_{n-1}} d t_{n} \sum_{j_{1}} \sum_{j_{2} \ldots j_{n}} \mathrm{e}^{-i\left(t-t_{1}\right) E_{1}}\left\langle j|V| j_{1}\right\rangle \times \\
& \times \mathrm{e}^{-i\left(t_{1}-t_{2}\right) E_{h_{1}}\left\langle j_{1}|V| j_{2}\right\rangle \ldots\left\langle j_{n-1}|V| j_{n}\right\rangle \mathrm{e}^{-i t_{n} E_{l_{n}}} d_{j_{n} .} .}
\end{aligned}
$$

Здесь предполагается, что конечное состояние не совпадает с начальным $\left(d_{j}=0\right)$, переменные интегрирования $t_{1}, t_{2}, \ldots, t_{n}$ имеют смысл времени переходов амплитуды состояния системы из одного собственного состояния гамильтониана $H_{(0)}$ в другое, а разности $t-t_{1}, t_{1}-t_{2}, \ldots$ $\ldots, t_{n-1}-t_{n}$ и $t_{n}-t_{0}$ определяют время задержки амплитуды состояния в состояниях $|j\rangle,\left|j_{1}\right\rangle, \ldots,\left|j_{n-1}\right\rangle$ и $\left|j_{n}\right\rangle$ соответственно.

Полученную формулу можно использовать, когда достаточно мало не только взаимодействие $V$, но и время $t-t_{0}$, поскольку слагаемые с $E_{j_{h}}=E_{j_{k^{\prime}}}\left(k \neq k^{\prime}\right)$ при $t-t_{0} \rightarrow \infty$ расходятся. В случае дискретного спектра эти слагаемые могут быть опущены, так как они компенсируют друг друга [ $\left.{ }^{21}\right]$. Однако при непрерывном (квазинепрерывном) спектре энергий (а именно такой спектр имеет система, способная к необратимому развитию во времени - релаксации) простое вычеркивание таких членов не устраняет расходимостей ввиду того, что в сумме (4) остаются члены со сколь угодно близкими энергиями. Поэтому в формуле (4) перейти к интересующему нас пределу $t_{0} \rightarrow-\infty$ нельзя.

Причина расходимостей слагаемых в разложении (4) при $t-t_{0} \rightarrow \infty$ связана с тем, что оно написано без учета затухания собственных состояний нулевого гамильтониана. Если указанное затухание учесть (см. Приложение), то $c_{j}(t)$ можно приближенно представить в виде

$$
\begin{aligned}
c_{j}(t) \approx & \sum_{n}(-i)^{n} \sum_{j_{1}} \sum_{j_{2} \ldots j_{n}}^{\prime} \int_{t_{0}}^{t} d t_{1} \int_{t_{0}}^{t_{1}} d t_{2} \ldots \int_{t_{0}}^{t_{n-1}} d t_{n} \mathrm{e}^{-i\left(E_{f}-i \gamma_{j}\right)\left(t-t_{1}\right)}\left\langle j|V| j_{1}\right\rangle \times \\
& \times \mathrm{e}^{-i\left(E_{j_{1}}-i \gamma_{j_{1}}\right)\left(t_{1}-t_{2}\right)}\left\langle j_{1}|V| j_{2}\right\rangle \ldots\left\langle j_{n-1}|V| j_{n}\right\rangle \mathrm{e}^{-i E_{j_{n}} t_{n}} d_{j_{n}},
\end{aligned}
$$


где $\gamma_{j}-$ константа затухания состояния $|j\rangle$ (выражение для $\gamma_{j}$ см. в Приложении). Эта формула может быть использована при любых $t_{0}$, включая и $-\infty$, если только взаимодействие $V$ достаточно мало.

Рассмотрим теперь конкретно процесс преобразования падающих на центр первичных фотонов во вторичные. Гамильтониан $H_{(0)}$ в этом случае равен сумме гамильтонианов электромагнитного поля $\left(H_{R}\right)$ и примесного центра кристалла $\left(H_{I}\right)$

$$
H_{(0)}=H_{R}+H_{I},
$$

a собственные состояния $H_{(0)}$ равны произведению их собственных состояний

$$
|j\rangle=|N\rangle|l\rangle
$$

$(|N\rangle$ - состояние поля с $N$ фотонами, $|l\rangle$ - состояние примесного центра).

Гамильтониан взаимодействия электромагнитного поля и электронов примесного центра имеет вид

$$
V=-\frac{e}{m} \sum_{k} \mathrm{p}_{k} \mathrm{~A}_{k}
$$

Здесь $\mathbf{A}_{k}$ - векторный потенциал электромагнитного поля, действующий на $k$-й электрон, импульс которого $\mathrm{p}_{k}, e$ и $m-$ заряд и масса электрона. ** Матричные элементы этого гамильтониана взаимодействия на состояниях (7) отличны от нуля только для однофотонных переходов. Именно поэтому процесс исчезновения одного фотона и возникновения другого описывается не ниже, чем во втором порядке теории возмущений.

В данной работе мы ограничимся рассмотрением слабого возбуждающего поля, когда напряженность поля световой волны гораздо меньше напряженности внутриатомных полей. В этом случае для описания двухфотонного процесса можно ограничиться членом второго порядка в разложении (5)

$$
\begin{gathered}
c_{1 \Omega, l}(t) \approx-\int_{-\infty}^{t} d t_{1} \int_{-\infty}^{t_{1}} d t_{2} \sum_{l_{1} l_{2}}\left\langle l\left|v_{\Omega}\right| l_{1}\right\rangle\left\langle l_{1}\left|v_{\omega_{0}}\right| l_{2}\right\rangle \exp \left[-i\left(\Omega+E_{l}-i \gamma_{l}\right)\left(t-t_{1}\right)-\right. \\
\left.-i\left(E_{l_{1}}-i \gamma_{l_{1}}\right)\left(t_{1}-t_{2}\right)-i E_{l_{2}} t_{2}\right] s\left(t_{2}\right) d_{l_{2}}
\end{gathered}
$$

где функция

$$
s(x)=\int d \omega \mathrm{e}^{-i \omega x} d_{\omega}
$$

определяет зависимость от времени возмущения, действующего со стороны возбуждающего света на центр, $\omega_{0}$ - средняя частота возбуждающего светового пакета (предполагается, что спектральная ширина возбуждающего пакета мала по сравнению с его средней частотой),

$$
\begin{aligned}
& v_{\omega_{0}}=\left\langle N-1_{\omega_{0}}|V| N\right\rangle, \\
& v_{\Omega}=\left\langle N-1_{\omega_{0}}+1_{\Omega}|V| N-1_{\omega_{0}}\right\rangle
\end{aligned}
$$

- однофотонные матричные элементы уничтожения фотона частоты $\omega_{0}$

** Гамильтониан взаимодействия $V$ содержит также член $\sim A_{k}^{2}$. Этот член описывает рождение и уничтожение двух одинаковых фотонов. В рассматриваемой задаче он не существен. 
и рождения фотона частоты $\Omega$. В формуле (9) учитываются лишь процессы, в которых в промежуточном состоянии на один фотон меньше, чем в исходном состоянии. Строго говоря, следует учитывать также процессы, в которых в промежуточном состоянии на один фотон больше, чем в исходном состоянии. Однако при резонансном возбуждении они несущественны [ $\left.{ }^{20}\right]$.

Выше была найдена амплитуда вероятности двухфотонного перехода из заданного состояния примесного центра, и электромагнитного поля в фиксированное состояние центра и поля. Однако на эксперименте обычно непосредственно регистрируется лишь вторичный фотон. Поэтому в дальнейшем будем рассматривать вероятность возникновения к моменту времени $t$ вторичного фотона заданной частоты (и поляризации) и не будем интересоваться нерегистрируемым конечным состоянием центра. Учтем также, что в действительности всегда приходится иметь дело с большим числом центров и излучателей, генерирующих возбуждающий свет. В таком случае для нахождения искомой вероятности следует $\left|c_{1 \Omega, l}(t)\right|^{2}$ просуммировать по конечным состояниям примесного центра $l$ и усреднить по начальным состояниям примесного центра и излучателей. Поскольку примесные центры можно считать находящимися в состоянии теплового равновесия, то $\left\langle d_{l_{2}} d_{l_{2}^{\prime}}\right\rangle_{I}=\delta_{l_{2}}, l_{2}^{\prime} \cdot n_{l_{2}}$, где $n_{l_{2}}=z^{-1} \mathrm{e}^{-E_{l_{2} / k T}}, z$ - статистическая сумма, $T$ - температура. Поэтому вероятность того, что к моменту времени $t$ будет излучен фотон частоты $\Omega$, равна

$$
\begin{gathered}
W(\Omega, t)=\sum_{u^{\prime}} n_{1} \sum_{l_{l^{\prime}}{ }_{1}}\left\langle l\left|v_{\omega_{0}}^{*}\right| l_{1}\right\rangle\left\langle l_{1}\left|v_{\Omega}^{*}\right| l^{\prime}\right\rangle\left\langle l^{\prime}\left|v_{\Omega}\right| l_{1}^{\prime}\right\rangle\left\langle l_{1}^{\prime}\left|v_{\omega_{0}}\right| l\right\rangle \\
\times \iint_{-\infty}^{t} d t_{1} d t^{\prime}{ }_{1} \int_{-\infty}^{t_{1}} d t_{2} \int_{-\infty}^{t_{1}^{\prime}} d t_{2}^{\prime} \exp \left\{i\left(E_{l_{1}}+i \gamma_{l_{1}}\right)\left(t_{1}-t_{2}\right)-i\left(\Omega+E_{l^{\prime}}\right)\left(t_{1}-t_{1}{ }_{1}\right)-\right. \\
\left.-\gamma_{l^{\prime}}\left(2 t-t_{1}-t_{1}^{\prime}\right)-i\left(E_{l^{\prime}}-i \gamma_{l^{\prime}}\right)\left(t_{1}^{\prime}{ }_{1}-t_{2}^{\prime}\right)+i E_{l}\left(t_{2}-t_{2}^{\prime}\right)\right\} S\left(t_{2}, t_{2}^{\prime}\right)
\end{gathered}
$$

Здесь

$$
S\left(t_{2}, t_{2}^{\prime}\right)=\left\langle s^{*}\left(t_{2}\right) s\left(t_{2}^{\prime}\right)\right\rangle_{R}
$$

- корреляционная функция возбуждающего электромагнитного поля, $\langle\ldots\rangle_{R}$ - знак усреднения по ансамблю излучателей $(R)$.

Полученная формула для конкретных расчетов сложна. Ее, однако, можно существенно упростить, если для состояний центра воспользоваться адиабатическим приближением, приняв, что как исходные $(|l\rangle)$, так и конечные $\left(\left|l^{\prime}\right\rangle\right)$ состояния относятся к основному $(|0\rangle)$ электронному уровню, а в качестве промежуточного учесть лишь один возбужденный (резонансный) электронный уровень $(|1\rangle)$ :

$$
\begin{array}{ll}
|l\rangle=|0\rangle|i\rangle, & \left|l^{\prime}\right\rangle=|0\rangle|f\rangle, \\
\left|l_{1}\right\rangle=|1\rangle|m\rangle, & \left|l^{\prime}{ }_{1}\right\rangle=|1\rangle\left|m^{\prime}\right\rangle .
\end{array}
$$

Здесь $|i\rangle$ и $|f\rangle$ - колебательные волновые функции основного электронного состояния, а $|m\rangle$ и $\left|m^{\prime}\right\rangle$ - колебательные волновые функции возбужденного электронного состояния. Тогда

$$
W(\Omega, t)=\sum_{i f} n_{i} \sum_{m m^{\prime}}\left\langle i\left|M_{1}\right| m\right\rangle\left\langle m\left|M_{2}\right| f\right\rangle\left\langle f\left|M_{2}^{*}\right| m^{\prime}\right\rangle\left\langle m^{\prime}\left|M_{1}\right| i\right\rangle \times
$$




$$
\begin{aligned}
X \int_{-\infty}^{t} d t_{1} d t^{\prime}{ }_{1} \int_{-\infty}^{t_{1}} d t_{2} \int_{-\infty}^{t_{1}^{\prime}} d t^{\prime}{ }_{2} \exp \left\{i\left(E_{m}+i \gamma_{m}\right)\left(t_{1}-t_{2}\right)+i\left(\Omega+E_{f}\right)\left(t_{1}^{\prime}-t_{1}\right)-\right. \\
\left.-i\left(E_{m^{\prime}}-i \gamma_{m^{\prime}}\right)\left(t_{1}^{\prime}{ }_{1}-t_{2}^{\prime}\right)+i E_{i}\left(t_{2}-t_{2}^{\prime}\right)\right\} S\left(t_{2}, t_{2}^{\prime}\right)
\end{aligned}
$$

где учтено, что $\gamma_{f}=0$, поскольку основное электронное состояние не испытывает распада;

$$
\begin{aligned}
& M_{1}=\left\langle 1\left|v_{\omega}\right| 0\right\rangle, \\
& M_{2}^{*}=\left\langle 0\left|v_{\Omega}\right| 1\right\rangle
\end{aligned}
$$

- электронные матричные элементы для поглощения и излучения. Учтем, что

$$
\begin{array}{ll}
E_{i}|i\rangle=H_{0}|i\rangle, & E_{f}|f\rangle=H_{0}|f\rangle, \\
E_{m}|m\rangle=H_{1}|m\rangle, & E_{m^{\prime}}\left|m^{\prime}\right\rangle=H_{1}\left|m^{\prime}\right\rangle,
\end{array}
$$

где $H_{0}$ и $H_{1}$ - колебательные гамильтонианы основного и возбужденного электронных состояний, и воспользуемся свойством полноты состояний $|f\rangle,|m\rangle$ и $\left|m^{\prime}\right\rangle$ :

$$
\sum_{f}|f\rangle\left\langle f\left|=\sum_{m}\right| m\right\rangle\left\langle m\left|=\sum_{m^{\prime}}\right| m^{\prime}\right\rangle\left\langle m^{\prime}\right|=1 .
$$

Тогда формулу (15) можно привести к следующей инвариантной форме

$$
W(\Omega, t)=\iint_{-\infty}^{t} d t_{1} d t^{\prime}{ }_{1} \int_{-\infty}^{t_{1}} d t_{2} \int_{-\infty}^{t_{1}^{\prime}} d t^{\prime}{ }_{2} \mathrm{e}^{i \Omega\left(t^{\prime}-t_{1}\right)} C\left(t_{1}, t_{2}, t^{\prime}{ }_{1}, t^{\prime}{ }_{2}\right) S\left(t_{2}, t^{\prime}{ }_{2}\right),
$$

где четырехвременная корреляционная функция $C$ примесного центра равна

$$
\begin{aligned}
C\left(t_{1}, t_{2}, t^{\prime}{ }_{1}, t^{\prime}{ }_{2}\right) & =\left\langle M_{1}^{*} \mathrm{e}^{i\left(H_{1}+i \gamma\right)\left(t_{1}-t_{2}\right)} M_{2} \mathrm{e}^{i H_{0}\left(t^{\prime}-t_{1}\right)} \times\right. \\
& \times M_{2}^{*} \mathrm{e}^{-i\left(H_{1}-i \gamma\right)\left(t_{1}^{\prime}-t^{\prime}\right)} M_{1} \mathrm{e}^{\left.i H_{0}\left(t_{2}-t^{\prime}\right)_{2}\right)_{0}}
\end{aligned}
$$

Здесь $\langle\ldots\rangle_{0} \equiv z^{-1} \mathrm{Sp}\left(\mathrm{e}^{-H_{0} / k T} \ldots\right)$ - знак квантово-статистического усреднения по исходным колебательным состояниям, $\gamma$ - оператор затухания возбужденного состояния, определяемый соотношением

$$
\gamma|m\rangle=\gamma_{m}|m\rangle .
$$

Формула (18) была получена ранее [22].

Обычно на эксперименте интерес представляет не вероятность $W(\Omega, t)$, а скорость ее изменения

$$
\Pi(\Omega, t)=\frac{d}{d t} W(\Omega, t),
$$

описывающая скорость регистрации фотонов частоты $\Omega$ в единицу времени. Эта величина называется мгновенным, или переходным спектром. Регистрируемый вторичный спектр выражается через П $(\Omega, t)$ следующим образом:

$$
I(\Omega, t, \Delta t)=\int_{0}^{\Delta t} d x \Pi(\Omega, t+x),
$$

где $\Delta t-$ время регистрации спектра.

Отметим, что минимальное время регистрации спектра ограничено 
лишь условием $\Delta t \gg 1 / \Omega$. Практически, однако, это время может быть гораздо меньше характерного времени изменения П $(\Omega, t)$. Следовательно, мгновенный (переходный) спектр П $(\Omega, t)$ также можно считать измеряемым (более подробно о теории детектирования фотонов см., напр., в $\left.\left[{ }^{23}\right]\right)$.

Продифференцируем формулу (18) по $t$. Получим

$$
\Pi(\Omega, t)=2 \operatorname{Re} \iint_{-\infty}^{t} d t_{1} d t^{\prime}{ }_{2} \int_{-\infty}^{t_{1}} d t_{2} \mathrm{e}^{i \Omega\left(t-t_{1}\right)} C\left(t_{1}, t_{2}, t, t^{\prime}{ }_{2}\right) S\left(t_{2}, t^{\prime}{ }_{2}\right) .
$$

Перейдем к переменным интегрирования

$$
\begin{aligned}
& \mu=t-t_{1}, \\
& \tau=t-t^{\prime}{ }_{2}, \\
& \tau^{\prime}=t_{1}-t_{2} .
\end{aligned}
$$

Эти переменные имеют следующий физический смысл: $\tau$ и $\tau^{\prime}$ описывают время пребывания двух амплитуд состояния центра в возбужденном электронном состоянии, а $\mu$ соответствует разности времен двух амплитуд состояния в конечном электронном состоянии (т. е. $\mu$ описывает разность фаз двух амплитуд состояния в конечном электронном состоянии). В этих переменных интегрирования

$$
\Pi(\Omega, t)=2 \operatorname{Re} \iint_{0}^{\infty} \int d \mu d \tau d \tau^{\prime} \mathrm{e}^{i \Omega \mu} A\left(\mu, \tau, \tau^{\prime}\right) S\left(t-\mu-\tau^{\prime}, t-\tau\right),
$$

где

$$
\begin{gathered}
A\left(\mu, \tau, \tau^{\prime}\right) \equiv C\left(0,-\tau^{\prime}, \mu, \mu-\tau\right)= \\
=\left\langle M_{1}^{*} \mathrm{e}^{i \tau^{\prime}\left(H_{1}+i \gamma\right)} M_{2} \mathrm{e}^{i \mu H_{0}} M_{2}^{*} \mathrm{e}^{-i \tau\left(H_{1}-i \gamma\right)} M_{1} \mathrm{e}^{\left.-i\left(\mu+\tau^{\prime}-\tau\right) H_{0}\right\rangle_{0}} .\right.
\end{gathered}
$$

Учтем, что

$$
S^{*}\left(t-\mu-\tau^{\prime}, t-\tau\right)=S\left(t-\tau, t-\mu-\tau^{\prime}\right),
$$

а также, что

$$
\begin{aligned}
& \left.A^{*}\left(\mu, \tau, \tau^{\prime}\right)=\sum_{i f} n_{i} \sum_{m m^{\prime}}\left[\left\langle i\left|M_{1}^{*}\right| m\right\rangle\left\langle m\left|M_{2}\right| f\right\rangle\langle f| M_{2}^{*}\right] m^{\prime}\right\rangle\left\langle m^{\prime}\left|M_{1}\right| i\right\rangle \times \\
& \left.\times \exp \left\{i \tau^{\prime}\left(E_{m}+i \gamma_{m}\right)+i E_{f} \mu-i \tau\left(E_{m^{\prime}}-i \gamma_{m^{\prime}}\right)-i\left(\mu+\tau^{\prime}-\tau\right) E_{i}\right\}\right]^{*}= \\
& =\sum_{i f} n_{i} \sum_{m m^{\prime}}\left\langle i\left|M_{1}^{*}\right| m^{\prime}\right\rangle\left\langle m^{\prime}\left|M_{2}\right| f\right\rangle\left\langle f\left|M_{2}^{*}\right| m\right\rangle\left\langle m\left|M_{1}\right| i\right\rangle \times \\
& \times \exp \left\{-i \tau^{\prime}\left(E_{m}-i \gamma_{m}\right)-i E_{f} \mu+i \tau\left(E_{m^{\prime}}+i \gamma_{m^{\prime}}\right)+i\left(\mu+\tau^{\prime}-\tau\right) E_{i}\right\}= \\
& =\left\langle M_{1}^{*} \mathrm{e}^{i \tau\left(H_{1}+i \gamma\right)} M_{2} \mathrm{e}^{-i \mu H_{\mathrm{o}}} M_{2}^{*} \mathrm{e}^{-i \tau^{\prime}\left(H_{1}-i \gamma\right)} M_{1} \mathrm{e}^{i\left(\mu+\tau^{\prime}-\tau\right) H_{\mathrm{o}}}\right\rangle_{0},
\end{aligned}
$$

T. e.

$$
A^{*}\left(\mu, \tau, \tau^{\prime}\right)=A\left(-\mu, \tau^{\prime}, \tau\right)
$$

Тогда искомый переходный спектр примет вид

$$
\Pi(\Omega, t)=\Pi_{0}(\Omega, t)+\Pi_{1}(\Omega, t),
$$

где

$\Pi_{0}(\Omega, t)=\int_{-\infty}^{\infty} d \mu \int_{0}^{\infty} \int^{\infty} d \tau d \tau^{\prime} \mathrm{e}^{i \Omega \mu} A\left(\mu, \tau, \tau^{\prime}\right) S\left(t-\mu / 2-\tau^{\prime}, t+\mu / 2-\tau\right)$, 


$$
\begin{gathered}
\Pi_{1}(\Omega, t)=2 \operatorname{Re} \iint_{0}^{\infty} \int d \mu d \tau d \tau^{\prime} \mathrm{e}^{i \Omega \mu} A\left(\mu, \tau, \tau^{\prime}\right)\left[S\left(t-\mu-\tau^{\prime}, t-\tau\right)-\right. \\
\left.-S\left(t-\mu / 2-\tau^{\prime}, t+\mu / 2-\tau\right)\right] .
\end{gathered}
$$

Сделаем следующее замечание. Формулы (28)-(30) были нами выведены для невырожденных основного и возбужденного электронных состояний. Легко, однако, убедиться (см., напр., $\left.\left[{ }^{10,12,24}\right]\right)$, что эти формулы применимы и в случае вырождения этих состояний, если $H_{0}, H_{1}$, $\gamma, M_{1}$ и $M_{2}$ понимать как матрицы. Так, если основное состояние вырождено $m$-кратно, а возбужденное $-n$-кратно, то $H_{0}$ есть квадратная матрица $m$-го ранга, $H_{1}$ и $\gamma$ - квадратные матрицы $n$-го ранга, $M_{1}$ и $M_{2}$ - прямоугольные матрицы с $n$ рядами и $m$ столбцами, а $M_{1}{ }^{*}$ и $M_{2}{ }^{*}$ - сопряженные $M_{1}$ и $M_{2}$ матрицы с $n$ рядами и $m$ столбцами. Более того, при рассмотрении $H_{0}, H_{1}, \gamma, M_{1}$ и $M_{2}$ как матриц формулы (28) - (30) остаются справедливыми, когда вырождение основного и (или) возбужденного уровней частично или полностью снято.

\section{3. Стационарный спектр РВС}

Формулы (28)-(30) позволяют найти спектр РВС не только в зависимости от времени, но и в стационарных условиях возбуждения. Действительно, при стационарном возбуждении двухвременная корреляционная функция возбуждающего света зависит лишь от разности времен, т. е.

$$
S_{s t}\left(t-\mu / 2-\tau^{\prime}, t+\mu / 2-\tau\right)=S_{s t}\left(t-\mu-\tau^{\prime}, t-\tau\right)=S_{s t}\left(0, \mu+\tau^{\prime}-\tau\right) .
$$

Вследствие этого в стационарном случае $\Pi_{1}(\Omega, t)=0$ и

$$
\Pi(\Omega, t) \equiv \Pi(\Omega)=\int d \omega W(\Omega, \omega)\left|d_{\omega}\right|^{2},
$$

где $W(\Omega, \omega)$ - стационарный спектр РВС при возбуждении монохроматическим светом частоты $\omega$, определяемый формулой

$$
W(\Omega, \omega)=\int_{-\infty}^{\infty} d \mu \int_{0}^{\infty} \int d \tau d \tau^{\prime} \mathrm{e}^{i \Omega \mu-i \omega\left(\mu+\tau^{\prime}-\tau\right)} A\left(\mu, \tau, \tau^{\prime}\right),
$$

которая с учетом (17) может быть переписана в виде

$$
W(\Omega, \omega)=2 \pi \sum_{i f} n_{i}\left|\sum_{m} \frac{\left\langle f\left|M_{2}^{*}\right| m\right\rangle\left\langle m\left|M_{1}\right| i\right\rangle}{E_{i}+\omega-E_{m}+i \gamma_{m}}\right|^{2} \delta\left(E_{i}+\omega-E_{f}-\Omega\right) .
$$

Это - хорошо известная формула второго порядка, обычно используемая для описания резонансного рассеяния.

\section{4. Сглаженный переходный спектр РВС в случае больших стоксовых потерь}

Основываясь на полученных выше общих формулах, рассмотрим детально свойства переходных спектров РВС примесных центров кристаллов в следующих предположениях.

1. Имеются два электронных уровня (основной и возбужденный), которые считаются невырожденными.

2. Справедливо приближение Кондона: $M_{1}=$ const, $M_{2}=$ const. В таком случае константы затухания $\gamma_{m}$ возбужденных электронно- 
колебательных уровней $|m\rangle$ не зависят от номера колебательного уровня и оператор $\gamma$ является $c$-числом: $\gamma=$ const.

3. Колебания кристалла характеризуются слабым ангармонизмом.

4. При электронном переходе меняется лишь положение равновесия ядер. В этом случае гамильтониан $H_{1}$ связан с гамильтонианом $H_{0}$ унитарным преобразованием сдвига:

$$
H_{1}=\omega_{1}+\mathrm{e}^{\nabla} H_{0} \mathrm{e}^{-\nabla} \text {. }
$$

Здесь $\omega_{1}$ - частота чисто-электронного перехода, $\nabla-$ линейный оператор импульсного типа, равный

$$
\nabla=\frac{1}{\sqrt{2}} \sum_{j} \xi_{0 j}\left(a_{j}^{+}-a_{j}\right)
$$

где $\xi_{0 j}-$ сдвиг безразмерной нормальной координаты $\xi_{j}=\frac{1}{\sqrt{2}}\left(a_{j}^{+}+a_{j}\right)$ при электронном переходе, $a_{j}^{+}$и $a_{j}$ - операторы рождения и уничтожения фонона частоты $\omega_{j}$.

5. Взаимодействие оптических электронов центра с колебаниями сильное: $S=\frac{1}{2} \sum_{j} \xi_{0 j}^{2} \gg 1$ - безразмерные стоксовы потери гораздо больше единицы.

6. Двухвременная корреляционная функция возбуждающего света факторизована и имеет вид

$$
S\left(t_{1}, t_{2}\right)=S_{0} \exp \left\{i \omega_{0}\left(t_{1}-t_{2}\right)-\sigma\left(t_{1}^{2}+t_{2}^{2}\right)\right\}
$$

Спектральная ширина $\sigma^{1 / 2}$, определяющая длительность возбуждающего света, удовлетворяет условию

$$
\omega_{0} \gg \sigma^{1 / 2} \gg \bar{\omega}
$$

означающему, что длительность возбуждения гораздо больше среднего периода световой волны, но гораздо меньше среднего периода колебаний решетки $\left(\bar{\omega}=\sum_{j} \xi_{0 j}^{2} \omega_{j} / 2 S-\right.$ средняя частота колебаний $)$.

7. Рассматривается сглаженный переходный спектр, т. е. спектр, измеренный с разрешением $\Delta \Omega$, удовлетворяющим условию

$$
\Delta \Omega \gg \bar{\omega} \text {. }
$$

Предполагается также, что начальное время регистрации $t$ гораздо больше длительности возбуждения:

$$
t \gg \sigma^{-1 / 2}
$$

В этом случае уширение спектра, связанное с соотношением неопределенностей $t \Delta \Omega \geqslant 1$, мало по сравнению со спектральной шириной возбуждающего света (это уширение в формуле (24) учитывается автоматически). вид

В отмеченных приближениях корреляционная функция $A$ принимает

$$
A\left(\mu, \tau, \tau^{\prime}\right)=\left|M_{1} M_{2}\right|^{2} \mathrm{e}^{i \omega_{1}\left(\tau^{\prime}-\tau\right)-\gamma\left(\tau+\tau^{\prime}\right)} A_{0}\left(\mu, \tau, \tau^{\prime}\right)
$$




$$
\begin{aligned}
A_{0}\left(\mu, \tau, \tau^{\prime}\right) & =\left\langle\mathrm{e}^{\nabla} \mathrm{e}^{i \tau^{\prime} H_{0}} \mathrm{e}^{-\nabla} \mathrm{e}^{i \mu H_{0}} \mathrm{e}^{\nabla} \mathrm{e}^{-i \tau H_{0}} \mathrm{e}^{-\nabla} \mathrm{e}^{\left.-i\left(\mu+\tau^{\prime}-\tau\right) H_{0}\right\rangle_{0}}=\right. \\
& =\left\langle\mathrm{e}^{\nabla} \mathrm{e}^{-\nabla\left(\tau^{\prime}\right)} \mathrm{e}^{\nabla\left(\mu+\tau^{\prime}\right)} \mathrm{e}^{-\nabla\left(\mu+\tau^{\prime}-\tau\right)}\right\rangle_{0} .
\end{aligned}
$$

Здесь $\nabla(x)=\mathrm{e}^{i x H_{0}} \nabla \mathrm{e}^{-i x H_{0}}-$ оператор $\nabla$ в момент времени $x$ в представлении Гейзенберга. В приближении слабого ангармонизма этот коррелятор был вычислен ранее в [7]. Он равен

$$
\begin{gathered}
A_{0}\left(\mu, \tau, \tau^{\prime}\right)=\exp \left[g(\mu)+g\left(\tau^{\prime}\right)+g(-\tau)+g\left(\mu+\tau^{\prime}-\tau\right)-g\left(\mu+\tau^{\prime}\right)-\right. \\
-g(\mu-\tau)],
\end{gathered}
$$

где

$$
\begin{aligned}
g(x) & =\left\langle\nabla^{2}\right\rangle_{0}-\langle\nabla \nabla(x)\rangle_{0}= \\
& =\frac{1}{2} \sum_{j} \xi_{0 j}^{2}\left[\left(n_{j}+1\right)\left(\mathrm{e}^{i \omega_{j} x-\Gamma_{j}|x|}-1\right)+n_{j}\left(\mathrm{e}^{-i \omega_{j} x-\Gamma_{j}|x|}-1\right)\right]
\end{aligned}
$$

- логарифм вероятности Фурье-образа нормированных спектров поглощения $\chi(\omega)$ и люминесценции $J(\omega)$ :

$$
\begin{aligned}
& x(\omega)=(1 / 2 \pi) \int_{-\infty}^{\infty} d x \exp \left[i\left(\omega_{1}-\omega\right) x+g(x)\right], \\
& J(\omega)=(1 / 2 \pi) \int_{-\infty}^{\infty} d x \exp \left[i\left(\omega-\omega_{1}\right) x+g(x)\right],
\end{aligned}
$$

$n_{j}=\left[\exp \left(\omega_{j} / k T\right)-1\right]^{-1}-$ среднее число фононов частоты $\omega_{j}$ при температуре $T, \Gamma_{j}-$ константа ангармонического затухания фонона $j$. При $|x| \rightarrow \infty$ функция $g(x) \rightarrow-f_{0}, \quad$ где $f_{0}=\frac{1}{2} \sum_{j} \xi_{0 j}^{2}\left(2 n_{j}+1\right), \quad$ причем $\exp \left(-f_{0}\right)$ определяет вероятность чисто-электронной линии. Область времени $|x| \sim \Gamma^{-1}$, в которой функция $g(x)$ заметно отличается от $-f_{0}$, определяет характерное время установления теплового равновесия по колебаниям, если в начальный момент времени было создано возмущение в локальной области кристалла $\left[{ }^{25}\right]$.

В случае сглаженного переходного спектра, удовлетворяющего условию (37), актуальные значения $|\mu|$ в интегралах (29) и (30) малы:

$$
|\mu| \leqslant 1 / \Delta \Omega \ll 1 / \omega
$$

причем, согласно (36) и (38), $|\mu| \ll$.

Введем следующие переменные интегрирования:

$$
\begin{aligned}
& s=\tau^{\prime}-\tau \\
& t_{1}=\frac{\tau+\tau^{\prime}}{2}
\end{aligned}
$$

Тогда

$$
\begin{gathered}
\Pi(\Omega, t) \approx S_{0}\left|M_{1} M_{2}\right|^{2} 2 \operatorname{Re} \int_{0}^{\infty} d \mu \int_{0}^{\infty} d t_{1} \int_{-2 t_{1}}^{2 t_{1}} d s \mathrm{e}^{i \Omega \mu+i \omega_{1} s} A_{1}\left(\mu, s, t_{1}\right) \times \\
\times \exp \left[-2 \gamma t_{1}-i \omega_{0}(\mu+s)-2 \sigma\left(t-t_{1}\right)^{2}-\sigma(\mu+s)^{2} / 2-\right. \\
\left.-2 \sigma \mu\left(t-t_{1}\right)-\sigma \mu^{2} / 2\right],
\end{gathered}
$$


где

$$
\begin{aligned}
& A_{1}\left(\mu, s, t_{1}\right)=\exp \left\{g(\mu)+g(\mu+s)+g\left(s / 2+t_{1}\right)+\right. \\
& \left.+g\left(s / 2-t_{1}\right)-g\left(s / 2+\mu+t_{1}\right)-g\left(s / 2+\mu-t_{1}\right)\right\} .
\end{aligned}
$$

Как видно из определения (43), переменная интегрирования $s$ представляет собой разность времен двух амплитуд состояния в возбужденном электронном состоянии, а $t_{1}$ - среднее время для обеих амплитуд. Поэтому $s$ описывает разность фаз амплитуд состояния, а зависимость корреляционной функции $A_{1}\left(\mu, s, t_{1}\right)$ от $s$ - фазовую колебательную релаксацию в возбужденном электронном состоянии. Зависимость же $A_{1}\left(\mu, s, t_{1}\right)$ от $t_{1}$ описывает зависимость корреляционной функции от времени жизни системы в возбужденном состоянии, т. е. энергетическую колебательную релаксацию в этом состоянии.

Из (44) и (42) следует, что актуальные значения $s$ и $t_{1}$ следующие:

$$
\begin{gathered}
|s| \leqslant 1 /\left(\Delta \Omega+\sigma^{1 / 2}\right) \ll 1 \bar{\omega}, \\
t_{1} \sim t \gg|s| .
\end{gathered}
$$

Это означает, что в рассматриваемой области времен мы имеем дело с РВС, испускаемым после фазовой колебательной релаксации. Поэтому это РВС следует классифицировать как ГЛ либо, если $t \gg \Gamma^{-1}$, как ОЛ.

Из (42) и (46) следует также, что в интеграле по $s$ в формуле (44) пределы интегрирования можно заменить на $\pm \infty$, а фигурирующие в (45) g-функции разложить в ряд по степеням $\mu$ и $s$ и учесть несколько первых членов. Тогда с точностью до членов $\sim \mu^{2}, s^{2}$ включительно искомый сглаженный переходный спектр приведется к виду

$$
\begin{gathered}
\Pi(\Omega, t)=\sqrt{\frac{\pi}{2 \sigma} S_{0}\left|M_{1} M_{2}\right|^{2} \mathrm{e}^{-2 \gamma t} 2 \operatorname{Re} \int_{0}^{\infty} \int_{-\infty}^{\infty} d \mu d y \exp \left[i\left(\omega_{a}-\omega_{0}\right) y-\frac{\sigma}{2} y^{2}+\right.} \\
\left.+i\left(\Omega-\omega_{L}+2 \varphi_{1}(t)\right) \mu-\frac{m_{2}}{2}\left(\mu^{2}+y^{2}\right)+\mu y \varphi_{2}(t)\right] .
\end{gathered}
$$

Здесь $y=\mu+s$,

$$
m_{k}=\left.\frac{d^{k}}{d x^{k}} g(x)\right|_{x=0}, \quad k=1,2
$$

- семиинвариант $k$-го порядка спектров поглощения и люминесценции,

$$
\omega_{a}=\omega_{1}+m_{1}, \quad \omega_{L}=\omega_{1}-m_{1}
$$

- частоты максимумов спектров поглощения и люминесценции,

$$
\begin{aligned}
& \varphi_{1}(t)=\operatorname{Im} d g(t) / d t \leqslant m_{1}, \\
& \varphi_{2}(t)=\operatorname{Re} d^{2} g(t) / d t^{2} \leqslant m_{2},
\end{aligned}
$$

т. е. $2 \varphi_{1}(t)$ меньше или равно расстоянию между максимумами полос поглощения и ОЛ, а $\varphi_{2}(t)$ меньше или равно второму центральному моменту этих полос, причем эти функции заметно отличны от нуля при $t \lesssim \Gamma^{-1}$. В формуле (47) мы учли, что в рассматриваемом случае $t \gg \sigma^{-1 / 2}$

$$
\mathrm{e}^{-2 \sigma\left(\left(t-t_{1}\right)^{2}-\mu\left(t-t_{1}\right)\right)} \approx \sqrt{\frac{\pi}{2 \sigma}} \delta\left(t-t_{1}\right)
$$

и $\quad m_{2} \gg \sigma$. 
Интегралы по $\mu$ и $y$ в (47) вычисляются точно. В результате формула для сглаженного переходного спектра принимает окончательный вид

$$
\Pi(\Omega, t) \approx \frac{B}{\beta(t)} \exp \left[-2 \gamma t-\frac{(\Omega-\omega(t))^{2}}{2 \beta^{2}(t)}\right]
$$

где

$$
\omega(t)=\omega_{L}+2 \varphi_{1}(t)+\left(\omega_{0}-\omega_{a}\right) \varphi_{2}(t) /\left(m_{2}+\sigma\right)
$$

и

$$
\beta^{2}(t)=m_{2}-\varphi_{2}^{2}(t) /\left(m_{2}+\sigma\right)
$$

определяют зависимость от времени положения максимума и ширины спектра соответственно, а

$$
B=S_{0}\left|M_{1} M_{2}\right|^{2}\left[\frac{2 \pi^{3}}{\sigma\left(m_{2}+\sigma\right)}\right]^{1 / 2} \mathrm{e}^{-\frac{\left(\omega_{0}-\omega_{a}\right)^{2}}{2\left(m_{2}+\sigma\right)}} .
$$

Приведем для сравнения сглаженные нормированные спектры поглощения и ОЛ в рассмотренной модели

$$
\begin{aligned}
& x(\omega)=\left[\frac{1}{2 \pi m_{2}}\right]^{1 / 2} \mathrm{e}^{-\frac{\left(\omega-\omega_{\alpha}\right)^{2}}{2 m_{2}}}, \\
& J(\omega)=\left[\frac{1}{2 \pi m_{2}}\right]^{1 / 2} \mathrm{e}^{-\frac{\left(\omega-\omega_{L}\right)^{2}}{2 m_{2}}} .
\end{aligned}
$$

\section{5. Обсуждение}

Полученный выше сглаженный переходный спектр П $(\Omega, t)$ имеет форму гауссовой кривой, положение максимума и ширина которой зависят от времени (см. рис. 1). Отмеченные изменения во времени положения $\omega(t)$ и ширины $\beta(t)$ спектра носят характер затухающих осцилляций. Наиболее быстро $\omega(t)$ и $\beta(t)$ изменяются в области $t \leqslant \Gamma^{-1}$. В частности, за первую четверть периода колебаний максимум сдвигается от максимума спектра поглощения к максимуму спектра ОЛ, а изменение ширины спектра по порядку величины равняется $m_{2}^{1 / 2}$, причем ширина спектра тем больше, чем ближе положение максимума к $\omega_{L}$. При $t \gg \Gamma^{-1}$ имеем $\varphi_{1}(t) \approx 0, \varphi_{2}(t) \approx 0$, а $\Pi(\Omega, t) \approx$ const $\mathrm{e}^{-2 \gamma t} J(\Omega)$, т. е. при больших временах $t$, соответствующих окончанию колебательной релаксации, спектр П $(\Omega, t)$ переходит в спектр ОЛ, как это и должно быть.

Рассмотрим более подробно физические причины таких изменений переходного спектра во времени, учитывая, что после поглощения фотона центр оказывается в сильно возбужденном колебательном состоянии, в котором квантовые эффекты малы. Поэтому, как было показано в $\left[{ }^{17}\right]$ (см. также $\left[{ }^{26}\right]$ ), спектр РВС можно приближенно найти, основываясь на принципе Франка-Кондона, согласно которому электронный переход происходит мгновенно. Вследствие этого координаты и импульсы ядер при переходе сохраняются, а интенсивность излучения на частоте $\Omega$ определяется вероятностью найти систему в конфигурационной координате $q$, удовлетворяющей условию $v(q)=\Omega$, где $v(q)=$ 


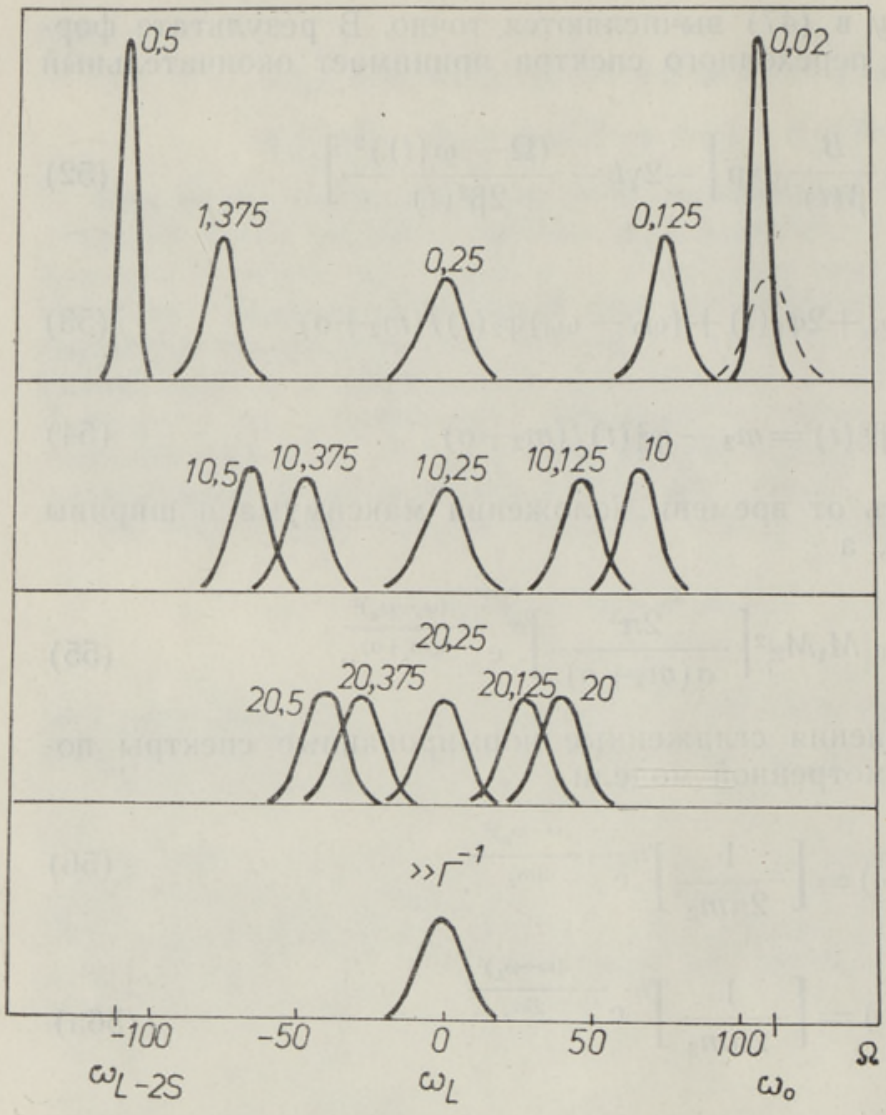

Рис. 1. Временное поведение переходных опектров П $(\Omega, t)$. Цифры вверху обозначают время $t$, измеренное в оредних периодах колебаний $\bar{T}=2 \pi / \bar{\omega}$; циф. ры внизу - безраз. мерные стоксовы потери $S$. Пунктиром воспроизведен спектр поглощения.

$=H_{1}-H_{0}$. В рассматриваемом случае $v(q)=\omega_{L}+q$, где $q=$ $=\sum_{j} \omega_{j} \xi_{0 j} \xi_{j}-$ симметризованная координата, отсчитанная от положения равновесия центра в возбужденном электронном состоянии. В начальный момент времени при резонансном возбуждении $\xi_{j} \approx \xi_{0 j}$, а $\xi_{j}(t) \approx \xi_{0 j} \cos \omega_{j} t \mathrm{e}^{-\mathrm{r}_{j} t}$. Поэтому в момент времени $t$ величина $q(t) \approx \sum_{j} \omega_{j} \xi_{0 j}^{2} \cos \omega_{j} t \mathrm{e}^{-\Gamma_{j} t} \approx 2 \varphi_{1}(t)$, вследствие чего максимум излучения в этот момент времени будет находиться на частоте $\Omega \approx \omega_{L}+2 \varphi_{1}(t)$. Это полностью согласуется с формулой (52), если учесть, что при резонансном возбуждении $\omega_{0}-\omega_{a} \approx 0$.

Таким образом, физическая причина осцилляционной зависимости от времени положения максимума сглаженного спектра РВС состоит в том, что конфигурационная координата $q$, приближенно определяющая частоту излучения, совершает в возбужденном электронном состоянии затухающие колебания.

Обсудим теперь зависимость ширины переходного спектра (52) от времени. Прежде всего заметим, что при $\Gamma<\bar{\omega}$ и $\sigma<m_{2}$ имеет место $\beta(t) \sim|d q(t) / d t|$, т. е. ширина спектра изменяется синхронно с изменением абсолютной величины скорости $v(t)=d q(t) / d t$ движения конфигурационной координаты $q$, причем она тем больше, чем больше $|v(t)|$. Такое изменение ширины спектра со временем легко понять, если учесть, что оптический переход в действительности происходит не мгновенно, а за время $\delta t \sim \sigma^{-1 / 2}$, что приводит к неопределенности на- 
чальной фазы колебания $\delta \varphi \sim \bar{\omega} \sigma^{-1 / 2}$. По этой причине при $t \gg \sigma^{-1 / 2}$ координата $q(t)$ приобретает неопределенность $\delta q(t) \sim \sigma^{-1 / 2}|d q(t) / d t|$, в результате чего и возникает поправка к ширине спектра, синхронно изменяющаяся с $|v(t)|$. Отметим также, что существенный вклад в ширину спектра вносят неопределенности частоты возбуждения и, тем самым, начальной координаты $\left(\delta q(0) \sim \sigma^{1 / 2}\right)$, а также неопределенность начальной скорости (в условиях теплового равновесия $\delta v(0) \sim$ $\sim(\bar{n}+1 / 2) \bar{\omega}^{2}$, где $\bar{n}-$ среднее значение $\left.n_{j}\right)$. Последняя приводит к дополнительной неопределенности начальной фазы колебаний в возбужденном состоянии, вследствие чего амплитуда изменений ширины спектра со временем увеличивается с ростом температуры.

\section{6. Сглаженный стационарный спектр РВС}

Выше речь шла конкретно о зависящем от времени сглаженном спектре РВС. Представляет интерес рассмотреть также сглаженный стационарный спектр РВС. В принятых приближениях этот спектр равен

$\Pi(\Omega)=\int_{-\infty}^{\infty} \Pi\left(\Omega, t-t^{\prime}\right) d t^{\prime} \approx B \int_{0}^{\infty} d t_{1} \beta^{-1}\left(t_{1}\right) \exp \left\{-2 \gamma t_{1}-\frac{\left(\Omega-\omega\left(t_{1}\right)\right)^{2}}{2 \beta^{2}\left(t_{1}\right)}\right\}$,

где переменная интегрирования $t_{1}$ имеет смысл времени жизни центра в возбужденном состоянии (в (57) учтено, что в предположениях (35) и (36) $\Pi(\Omega, t) \approx 0$, если $t \leqslant 0)$. Подчеркнем, что этот спектр содержит излучение не только после колебательной релаксации, т. е. ОЛ $\left(t_{1} \gg \Gamma^{-1}\right.$ в $\left.(57)\right)$, но и в процессе нее, т. е. ГЛ $\left(t_{1} \lesssim \Gamma^{-1}\right.$ в $\left.(57)\right)$. При $t_{1} \gg \Gamma^{-1}$ частота максимума излучения $\omega\left(t_{1}\right) \approx \omega_{L}$, а $\beta^{2}\left(t_{1}\right) \approx m_{2}$. Поэтому часть спектра (57), соответствующая ОЛ, описывается формулой

$$
\Pi_{L}(\Omega) \approx \frac{B}{2 \gamma m_{2}^{1 / 2}} \mathrm{e}^{-\frac{\left(\Omega-\omega_{L}\right)^{2}}{2 m_{2}}},
$$

которая с точностью до нормировочного множителя совпадает с хорошо известным выражением, определяемым формулой (56a).

Как было показано в $\left[{ }^{17}\right]$, стационарный спектр ГЛ имеет максимумы в области частот $\Omega=\omega\left(t^{(i)}\right)\left(t^{(i)}-\right.$ моменты времени классических точек поворота, являющиеся решениями уравнения $v(t)=0)$ :

$$
\Pi_{H L}(\Omega) \approx \sum_{i} \frac{2\left(1-\delta_{i, 0}\right)}{\sqrt{\left|z_{i}\right|}} \mathrm{e}^{-2 \gamma t^{(i)}} \Theta\left(z_{i} \operatorname{sign}\left(\omega\left(t^{(i)}\right)-\omega_{L}\right)\right),
$$

где $z_{i}=\Omega-\omega\left(t^{(i)}\right)$ (предполагается, что $\overline{S_{\omega}} \gg z_{i} \gg \bar{\omega}$ ). Отметим, что эта формула получается из (57), если перейти к пределу $\sigma \rightarrow 0$ (в этом случае в области точек поворота $\beta(t) \approx 0)$.

\section{7. Численные расчеты спектров $I(\Omega, t, \Delta t)$}

Как отмечалось выше, переходный спектр (52) заметно изменяется в области $\delta t \sim \bar{T} / 4 \sim 10^{-13}$ сек, где $\bar{T}=2 \pi / \bar{\omega}-$ средний период колебаний. Поэтому время регистрации этого спектра должно быть меньше $\bar{T} / 4$. На сегодняшний день необходимая для таких измерений экспериментальная техника еще не разработана. Поэтому представляет интерес 
провести расчеты спектров $I(\Omega, t, \Delta t)$ для времени регистрации $\Delta t \geqslant \bar{T}$. Для этого необходимо, однако, знать корреляционную функцию $g(t)$ (либо функции $\varphi_{1}(t)$ и $\left.\varphi_{2}(t)\right)$. Мы выберем эту функцию в виде

где

$$
g(t)=g_{c r}(t)+g_{l o c}(t),
$$

$$
g_{c r}(t)=S_{c r}\left[\frac{1}{\left(1-i_{\mathrm{Q}} t\right)^{2}}-1\right]
$$

учитывает взаимодействие оптических электронов с кристаллическими колебаниями $\left(\varrho=2 \bar{\omega}_{c r} / \sqrt{3} \cdot \pi \approx \bar{\omega}_{c r} / 3, \bar{\omega}_{c r}-\right.$ средняя частота кристаллических колебаний), а

$$
g_{l o c}(t)=S_{l o c}\left(\mathrm{e}^{i \omega_{\text {loc }} t-\Gamma|t|}-1\right)
$$

- с локальным (псевдолокальным) колебанием. Здесь считается, вопервых, что температура низкая, вследствие чего $\bar{n} \approx 0$, и, во-вторых, что константа затухания локального (псевдолокального) колебания Г гораздо меньше частоты его $\omega_{l o c}$. Отметим, что выбранная в таком виде функция $g(t)$ качественно правильно описывает поведение $g(t)$ в конкретных центрах при малых и больших $|t|$. Однако в области промежуточных $t$ оно может быть существенно иным.***
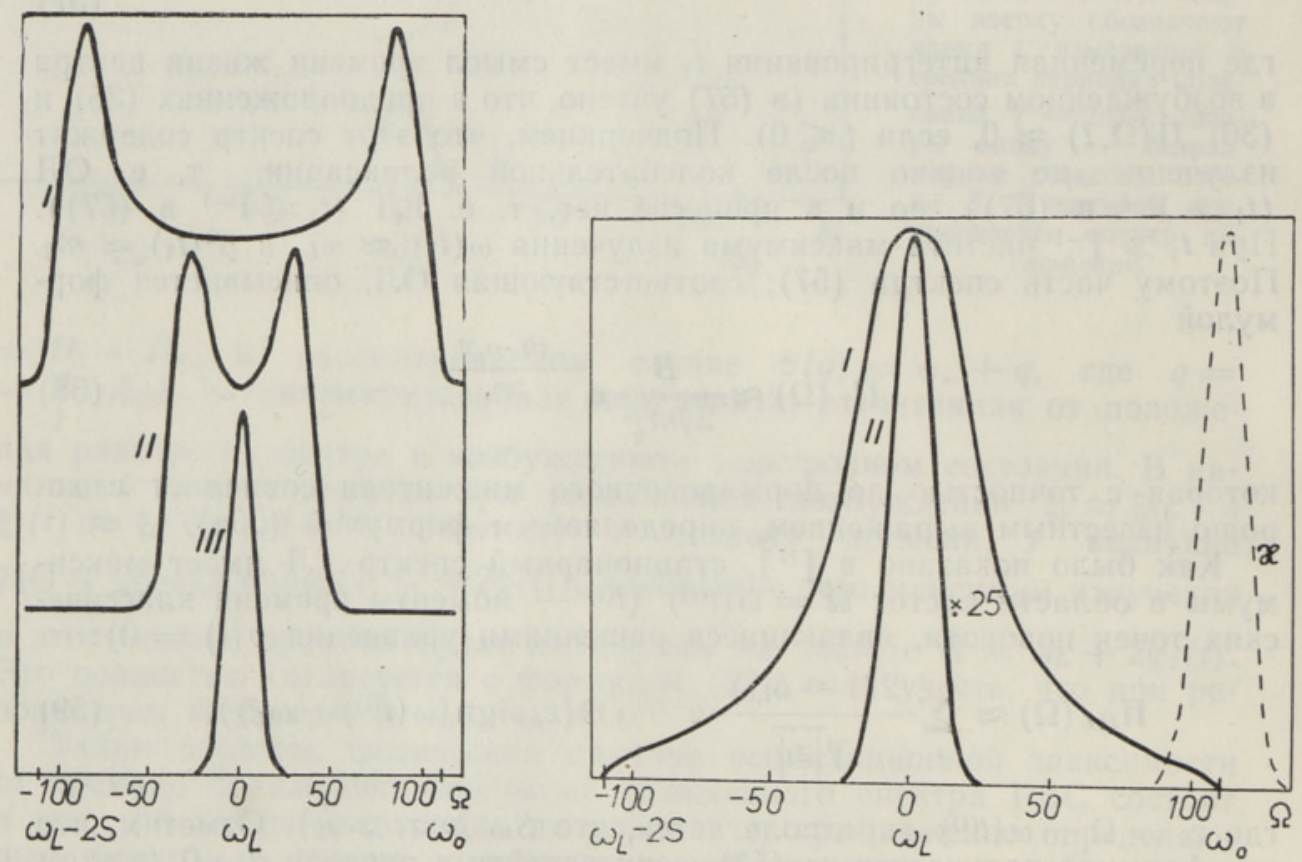

Рис. 2. Спектры $I(\Omega, t, \Delta t)$ с учетом лишь локального (псевдолокального) колебания. Константа затухания $\Gamma=1 / 20 T_{\text {loc }}=\omega_{\text {loc }} / 40 \pi$. $a-\frac{}{T}$ Время регистрации $\Delta t=5 T_{\text {loc; }}$ начальное время $t=0,04$ (I), 20 (II) и $400 T_{\text {loc }}$ (III). б- - Время регистрации $\Delta t=50 T_{\text {loc; }}$ начальное время $t=0,04$ (I) и $500 T_{\text {loc }}$ (II). $\not{x}-$ спектр поглощения.

*** Нахождение корреляционных функций типа $g(t)$ требует привлечения детальной информации о примесном центре и является задачей динамики кристаллических решеток. При этом для каждого центра указанная функция имеет свой конкретный вид. Поэтому выполненные в данной работе расчеты спектров $I(\Omega, t, \Delta t)$ с использованием модельных функций $(60)-(62)$ имеют лишь иллюстративное значение. 

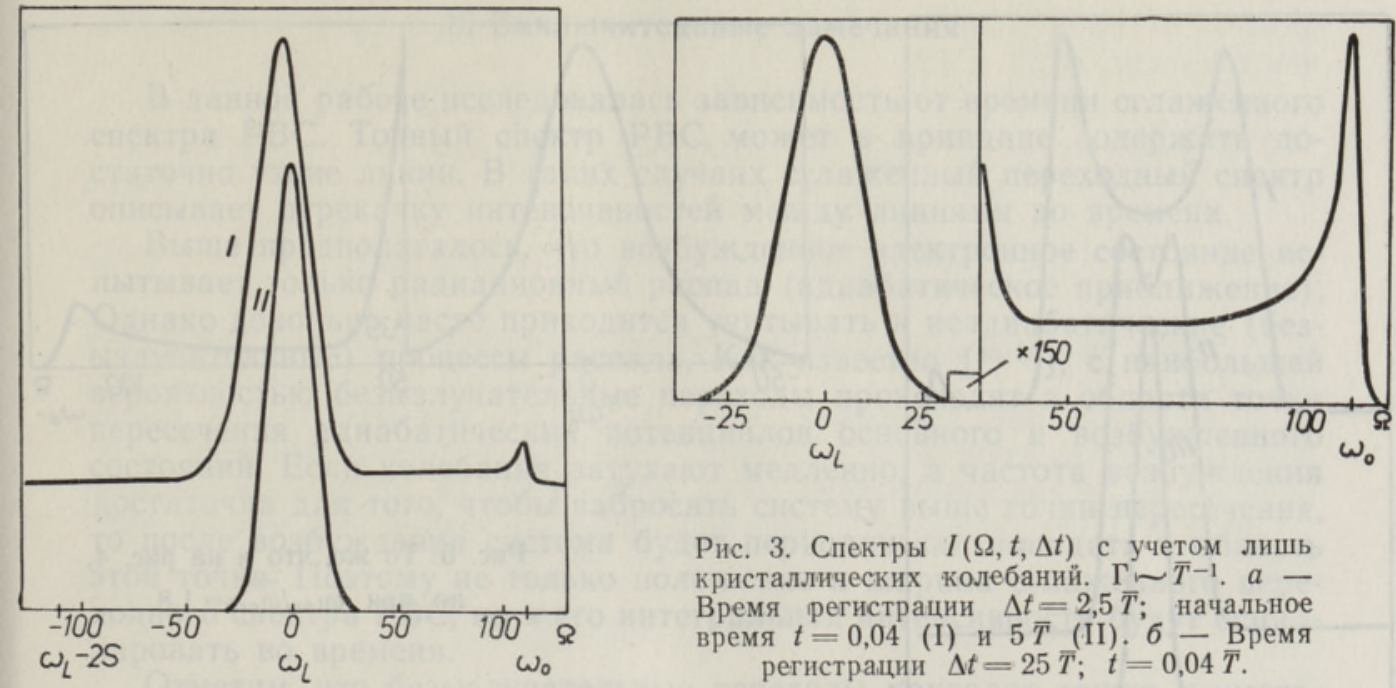

Рис. 3. Спектры $I(\Omega, t, \Delta t)$ с учетом лишь кристаллических жолебаний. $\Gamma \sim \bar{T}^{-1}, a-$ Время регистрации $\Delta t=2,5 \bar{T}$; начальное время $t=0,04$ (I) и $5 \bar{T}$ (II). $6-$ Время регистрации $\Delta t=25 \bar{T} ; t=0,04 \bar{T}$.
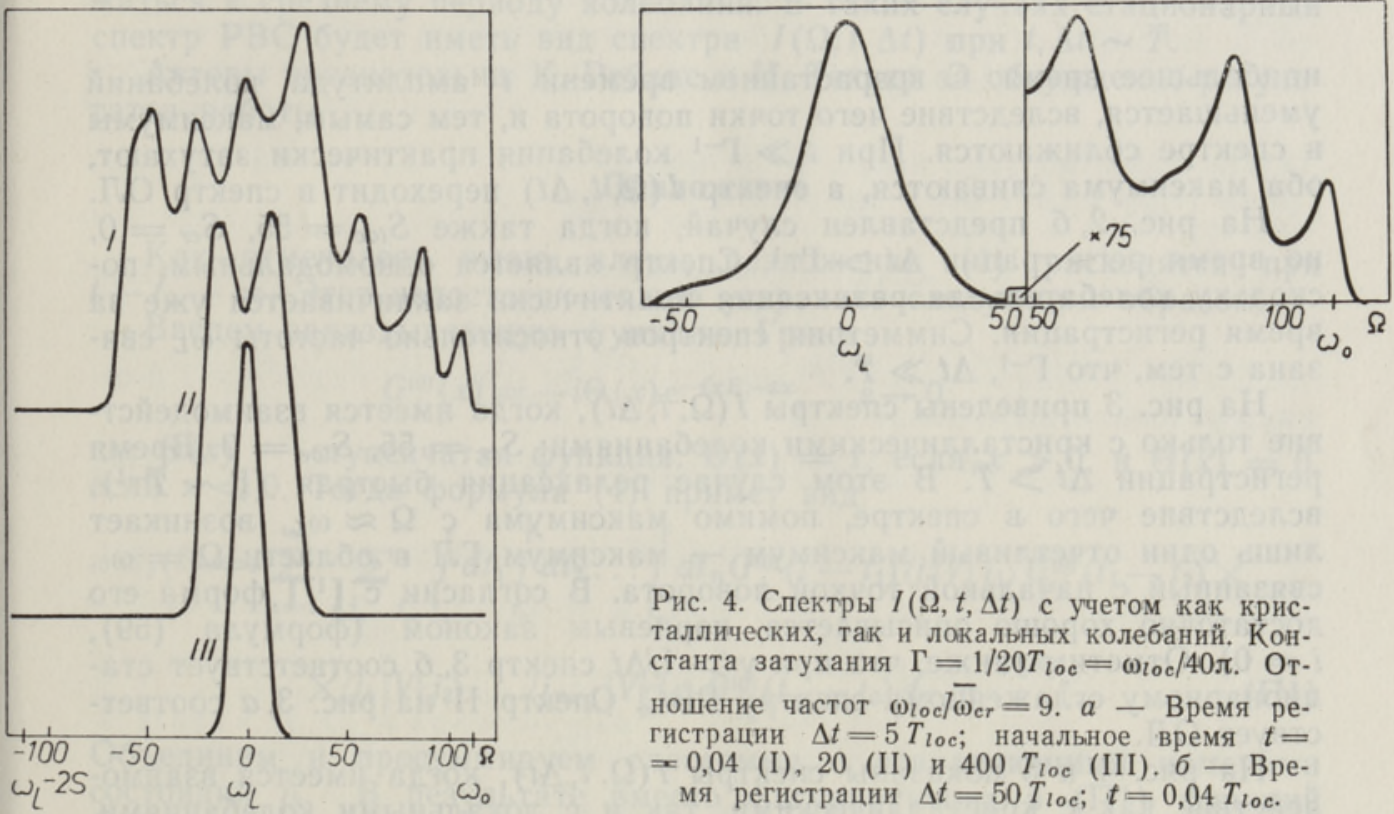

Рис. 4. Спектры $I(\Omega, t, \Delta t)$ с учетом как кристаллических, так и локальных колебаний. Константа затухания $\Gamma=1 / 20 T_{\text {loc }}=\omega_{\text {loc }} / 40 \pi$. Отношение частот $\omega_{l o c} / \bar{\omega}_{c r}=9 . a-$ Время регистрации $\Delta t=5 T_{\text {loc }}$; начальное время $t=$ $=0,04$ (I), 20 (II) и $400 T_{\text {loc }}$ (III). $6-$ Время регистрации $\Delta t=50 T_{\text {loc }} ; t=0,04 T_{\text {loc }}$.

Численные расчеты были выполнены на ЭВМ M-4030. Во всех случаях стоксовы потери были выбраны одинаковыми: $S=S_{l o c}+S_{\mathrm{cr}}=55$. Результаты расчетов приведены на рис. $2-5$.

Рис. 2, $a$ иллюстрирует взаимодействие лишь с медленно затухающим локальным (псевдолокальным) колебанием: $S_{l o c}=55, S_{c r}=0$. Время регистрации $\Delta t \ll \Gamma^{-1}$. На начальных этапах релаксации $\left(t \leqslant \Gamma^{-1}\right.$; случаи I и II) спектр имеет двумодальную форму, что согласуется с выводом работы $\left[{ }^{27}\right]$, основанным на анализе факторов Франка-Кондона. Максимумы в спектре обусловлены излучением центра из областей классических точек поворота, в которых система проводит 

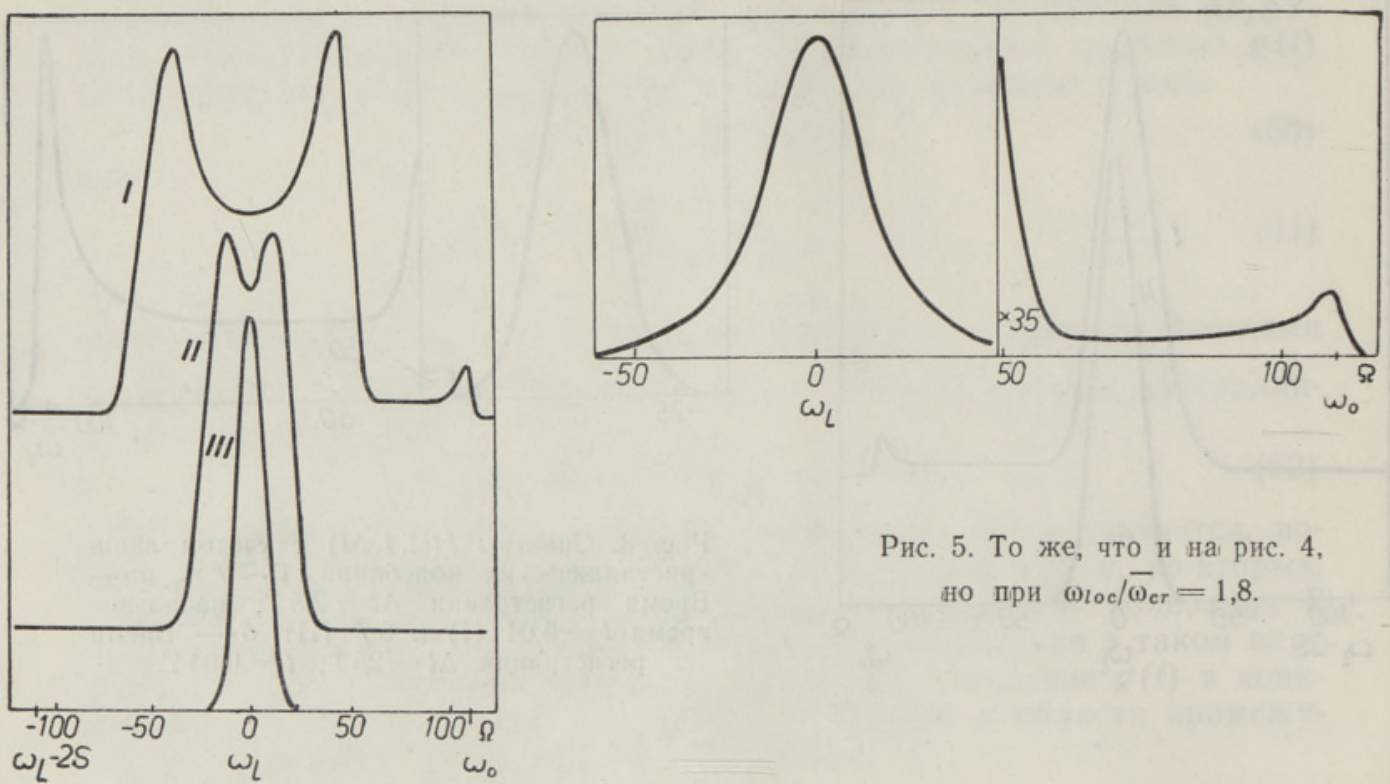

Рис. 5. То же, что и на рис. 4 , но при $\omega_{l o c} / \bar{\omega}_{c r}=1,8$.

наибольшее время. С возрастанием времени $t$ амплитуда колебаний уменьшается, вследствие чего точки поворота и, тем самым, максимумы в спектре сближаются. При $t \gg \Gamma^{-1}$ колебания практически затухают, оба максимума сливаются, а спектр $I(\Omega, t, \Delta t)$ переходит в спектр ОЛ.

На рис. 2, б представлен случай, когда также $S_{l o c}=55, S_{c r}=0$, но время регистрации $\Delta t>\Gamma^{-1}$. Спектр является одномодальным, поскольку колебательная релаксация практически заканчивается уже за время регистрации. Симметрия спектров относительно частоты $\omega_{L}$ связана с тем, что $\Gamma^{-1}, \Delta t \gg \bar{T}$.

На рис. 3 приведены спектры $I(\Omega, t, \Delta t)$, когда имеется взаимодействие только с кристаллическими колебаниями: $S_{c r}=55, S_{l o c}=0$. Время регистрации $\Delta t>\bar{T}$. В этом случае релаксация быстрая $\left(\Gamma \sim \bar{T}^{-1}\right)$, вследствие чего в спактре, помимо максимума с $\Omega \approx \omega_{L}$, возникает лишь один отчетливый максимум - максимум ГЛ в области $\Omega=\omega_{0}$, связанный с начальной точкой поворота. В согласии с $\left[{ }^{17}\right]$ форма его достаточно хорошо описывается корневым законом (формула (59), $i=0)$. Отметим также, что при $\gamma>1 / \Delta t$ спектр 3 , б соответствует стационарному сглаженному спектру РВС. Спектр II на рис. 3, $a$ соответствует ОЛ.

На рис. 4 и 5 показаны спектры $I(\Omega, t, \Delta t)$, когда имеется взаимодействие как с кристаллическими, так и с локальными колебаниями, причем $S_{l o c}=S_{c r}=27,5$. В случае малых $t$ максимумы в спектрах связаны с излучением из областей классических точек поворота затухающих колебаний. К началу измерения спектров II на рис. $4, a$ и $5, a$ релаксация по кристаллическим колебаниям уже закончилась, но локальное колебание еще не затухло, вследствие чего эти спектры, как и спектры I и II на рис. 2, $a$, имеют двумодальную форму. Спектры III на рис. $4, a$ и $5, a$ испускаются после окончания релаксации по всем колебаниям, т. е. соответствуют ОЛ. 


\section{8. Заключительные замечания}

В данной работе исследовалась зависимость от времени сглаженного спектра РВС. Точный спектр РВС может в принципе содержать достаточно узкие линии. В таких случаях сглаженный переходный спектр описывает перекачку интенсивностей между линиями во времени.

Выше предполагалось, что возбужденное электронное состояние испытывает только радиационный распад (адиабатическое приближение). Однако довольно часто приходится учитывать и неадиабатические (безызлучательные) процессы распада. Как известно $\left[{ }^{28,29}\right]$, с наибольшей вероятностью безызлучательные переходы происходят в области точки пересечения адиабатических потенциалов основного и возбужденного состояний. Если колебания затухают медленно, а частота возбуждения достаточна для того, чтобы забросить систему выше точки пересечения, то после возбуждения система будет периодически попадать в область этой точки. Поэтому не только положение и ширина сглаженного переходного спектра РВС, но и его интегральная интенсивность будут осциллировать во времени.

Отметим, что безызлучательные переходы приводят также к уменьшению времени жизни $\gamma^{-1}$ равновесного по колебаниям возбужденного состояния. При высоких температурах указанное время может приближаться к среднему периоду колебания. В таких случаях стационарный спектр РВС будет иметь вид спектра $I(\Omega, t, \Delta t)$ при $t, \Delta t \sim \bar{T}$.

Авторы признательны К. Ребане и И. Техвер за обсуждение результатов работы.

\section{Приложение}

Как отмечалось выше, члены разложения (4) расходятся при $t-t_{0} \rightarrow \infty$. Этот недостаток можно устранить следующим образом.

Введем запаздывающую функцию Грина

$$
G_{j}^{(0)}(x)=-i \Theta(x) \mathrm{e}^{-i x E_{f}-\varepsilon x}, \quad \varepsilon \rightarrow 0,
$$

где $\Theta(x)$ - ступенчатая функция: $\Theta(x)=1$, если $x>0$, и $\Theta(x)=0$, если $x<0$. Тогда формула (4) примет вид

$$
\begin{gathered}
c_{j}(t)=i \sum_{n=1}^{\infty} \sum_{j_{1} j_{2} \ldots j_{n}} \int_{t_{0}}^{t} d t_{1} \int_{t_{0}}^{t} d t_{2} \ldots \int_{t_{0}}^{t} d t_{n} G_{j}^{(0)}\left(t-t_{1}\right)\left\langle j|V| j_{1}\right\rangle G_{j_{1}}^{(0)}\left(t_{1}-t_{2}\right) \times \\
\times\left\langle j_{1}|V| j_{2}\right\rangle \ldots\left\langle j_{n-1}|V| j_{n}\right\rangle G_{j_{n}}^{(0)}\left(t_{n}-t_{0}\right) d_{j_{n}} \mathrm{e}^{-i t_{0} E_{j_{n}}} .
\end{gathered}
$$

Объединим и просуммируем слагаемые с совпадающими номерами состояний $j_{k}$. В результате вместо фигурирующих в (П1) функций $G_{j}^{0}(x)$ возникнут точные запаздывающие функции Грина

$$
G_{j}(x)=-i \Theta(x)\left\langle j\left|\mathrm{e}^{-i x H-\varepsilon x}\right| j\right\rangle, \quad \varepsilon \rightarrow 0,
$$

а в сумме останутся лишь члены с несовпадающими состояниями:

$$
\begin{gathered}
c_{j}(t)=i \sum_{n=1}^{\infty} \sum_{j_{1} j_{2} \ldots j_{n}}^{\prime} \int_{t_{0}}^{t} d t_{1} \int_{t_{0}}^{t} d t_{2} \ldots \int_{t_{0}}^{t} d t_{n} G_{j}\left(t-t_{1}\right)\left\langle j|V| j_{1}\right\rangle G_{j_{1}}\left(t_{1}-t_{2}\right) \times \\
\times\left\langle j_{1}|V| j_{2}\right\rangle \ldots\left\langle j_{n-1}|V| j_{n}\right\rangle G_{j_{n}}\left(t_{n}-t_{0}\right) d_{j_{n}} \mathrm{e}^{-i t_{0} E_{j_{n}}}
\end{gathered}
$$

(штрих означает, что члены с совпадающими номерами состояний опу- 
щены). В справедливости формулы (ПЗ) легко убедиться, если учесть, что согласно (П2) и (3)

$$
\begin{gathered}
G_{j}(x)=G_{j}^{(0)}(x)+\sum_{n=1}^{\infty} \sum_{j_{1} j_{2} \ldots j_{n}} \delta_{j, j_{n}} \int_{0}^{x} d x_{1} \int_{0}^{x} d x_{2} \ldots \int_{0}^{x} d x_{n} G_{j}^{(0)}\left(x-x_{1}\right)\left\langle j|V| j_{1}\right\rangle \times \\
\times G_{j_{1}}^{(0)}\left(x_{1}-x_{2}\right)\left\langle j_{1}|V| j_{2}\right\rangle \ldots\left\langle j_{n-1}|V| j_{n}\right\rangle G_{j_{n}}^{(0)}\left(x_{n}\right),
\end{gathered}
$$

т. е. сумма членов с совпадающими номерами состояний действительно дает $G_{j}$.

В задачах типа рассеяния, РВС и других, как отмечалось выше, можно считать известным состояние системы в момент времени $t_{0}=$ $=-\infty$, когда взаимодействие выключено. В таком случае для фигурирующих в (П3) функций Грина исходных состояний $\left|j_{n}\right\rangle$ можно принять

$$
G_{j_{n}}\left(t-t_{0}\right)=G_{j_{n}}^{(0)}\left(t-t_{0}\right), \quad t_{0} \rightarrow-\infty
$$

Әто дает

$$
\begin{gathered}
c_{j}(t)=i \sum_{n=1}^{\infty} \sum_{j_{1} j_{2} \ldots j_{n} \rightarrow \infty}^{\prime} \int_{-\infty}^{t} d t_{1} \int_{-\infty}^{t} d t_{2} \ldots \int_{-\infty}^{t} d t_{n} G_{j}\left(t-t_{1}\right)\left\langle j|V| j_{1}\right\rangle \times \\
\times G_{j_{1}}\left(t_{1}-t_{2}\right)\left\langle j_{1}|V| j_{2}\right\rangle \ldots\left\langle j_{n-1}|V| j_{n}\right\rangle \mathrm{e}^{-i E_{j_{n}}{ }^{t}{ }{ }} d_{j_{n} \cdot}
\end{gathered}
$$

Как известно (см., напр., $\left.\left[{ }^{30}\right]\right)$, Фурье-образ $G_{j}(\omega)$ точной функции Грина (П2) может быть представлен в виде

$$
G_{j}(\omega)=\frac{1}{\omega-\omega_{j}-\Sigma_{j}(\omega)},
$$

где $\Sigma_{j}(\omega)$ - поляризационный оператор, для которого существует формально точное выражение

$$
\Sigma_{j}(\omega)=\left\langle j\left|V \lambda_{j} G_{\lambda_{j}} \lambda_{j} V\right| j\right\rangle .
$$

Здесь $\lambda_{j}$ - оператор проектирования, обладающий следующими свойствами:

$$
\begin{aligned}
& \lambda_{j}=1-|j\rangle\langle j|, \\
& \lambda_{j}|j\rangle=0, \quad \lambda_{j}^{2}=\lambda_{j},
\end{aligned}
$$

a

$$
G_{\lambda_{j}}(\omega)=\frac{1}{\omega-H_{0}-\lambda_{j} V \lambda_{j}+i \varepsilon}, \quad \varepsilon \rightarrow 0 .
$$

В случае непрерывного (квазинепрерывного) спектра поляризационный оператор имеет конечные как действительную, так и мнимую части. Следовательно, при возрастании $x$ точные функции Грина $G_{j}(x)$ затухают с конечной скоростью. Вследствие этого члены ряда (ПЗ) остаются конечными при сколь угодно больших $t-t_{0}$. В результате радиус сходимости ряда (П6) определяется величиной взаимодействия.

Если взаимодействие мало, то в формуле (П6) можно учесть лишь первое отличное от нуля слагаемое, а поляризационный оператор вычислить приближенно по формуле 


$$
\begin{gathered}
\sum_{j}(\omega) \approx\left\langle j\left|V \lambda_{j} G^{(0)}(\omega) \lambda_{j} V\right| j\right\rangle= \\
=\sum_{j_{1}}\left|\left\langle j|V| j_{1}\right\rangle\right|^{2}\left(\omega-E_{j_{1}}+i \varepsilon\right)^{-1}, \quad \varepsilon \mapsto 0 .
\end{gathered}
$$

Здесь

$$
G^{(0)}(\omega)=\frac{1}{\omega-H_{0}+i \varepsilon}, \quad \varepsilon \rightarrow 0 .
$$

Учтем, что в (П7) актуальные значения $\omega \approx E_{j}$. Поэтому в (П8) $\omega$ можно заменить на $E_{j}$. Тогда

$$
\Sigma_{j}(\omega) \approx \Delta E_{j}+i \gamma_{j}
$$

где

$$
\begin{gathered}
\Delta E_{j}=P \sum_{j_{1}}^{\prime}\left|\left\langle j|V| j_{1}\right\rangle\right|^{2}\left(E_{j}-E_{j_{1}}\right)^{-1}, \\
\gamma_{j}=\sum_{j_{1}}^{\prime}\left|\left\langle j|V| j_{1}\right\rangle\right|^{2} \delta\left(E_{j}-E_{j_{1}}\right)
\end{gathered}
$$

( $P$ - символ главного значения). Учет $\Delta E_{j}$ приводит к небольшой поправке к положению уровней, которой можно пренебречь; учѐт же $\gamma_{j}$ существен, т. к. $\gamma_{j}$ определяет скорость затухания функции Грина.

Таким образом, если взаимодействие $V$ мало, то в разложении (П6) можно учесть первое отличное от нуля слагаемое, а для функций Грина использовать приближение

$$
G_{j}(x) \approx-i \Theta(x) \mathrm{e}^{-i E_{j} x-i \gamma_{j} x},
$$

отличающееся от $G_{j}^{(0)}(x)$ лишь наличием затухания. В этом приближении

$$
\begin{aligned}
c_{j}(t) \approx & \sum_{n}(-i)^{n} \sum_{j_{1} j_{2} \ldots j_{n}} \sum_{-\infty}^{\prime} \int_{-\infty}^{t} d t_{1} \int_{-\infty}^{t_{1}} d t_{2} \ldots \int_{-\infty}^{t_{n-1}} d t_{n} \mathrm{e}^{-i\left(E_{f}-i \gamma_{j}\right)\left(t-t_{1}\right)}\left\langle j|V| j_{1}\right\rangle \mathrm{X} \\
& \times \mathrm{e}^{-i\left(E_{f_{1}}-i \gamma_{f_{1}}\right)\left(t_{1}-t_{2}\right)}\left\langle j_{1}|V| j_{2}\right\rangle \ldots\left\langle j_{n-1}|V| j_{n}\right\rangle \mathrm{e}^{-i E_{f_{n}} t_{n} d_{j_{n}} .}
\end{aligned}
$$

\section{Л ИТЕРА Т У Р А}

1. Rentzepis, P. M., Top p, M. R., Jones, R. P., Jorner, J., Phys. Rev. Lelt., $25,1742(1970)$.

2. Alf a no, R. R., Sha piro, S. L., Phys. Rev. Lett., 26, 1247 (1971).

3. La uberea u, A., Ka i se r, W., Opto-Electronics, 6, 1 (1974).

4. Песин М. С., Фабелинский И. Л., УФН, 120,273 (1976).

5. L a u bere a u, A., In: Proc. 5th Intern. Conf. on Raman Spectrosc., Freiburg, 1976, p. 353.

6. Электроника (пер. с англ.), 49, № 2, 106 (1976).

7. Техв ер И., Х и жняков В., Изв. АН ЭССР, Сер. физ.-матем. и техн. наук, 15, 9 (1966).

8. Hizhnyakov, V., Tehver, I., Phys. stat. sol., 21, 755 (1967); 39, 67 (1970).

9. Rebane, K. K., Tehver, I. J., Hizhnyakov, V. V., In: Proc. 1st SovietAmerican Symposium, Moscow, 1976, p. 467.

10. Hizhnyakov, V. V., Tehver, I. J., In: Proc. 2nd Intern. Conf. ed. M. Balkanski, Paris, 1971, p. 57.

11. Rebane, K., Hizhnyakov, V., Tehver, I., Изв. АН ЭССР, Физ. Матем., 16, 207 (1967).

12. Ребане К. К., Хи жняков В. В., Препринт ИФА-28, Тарту, 1973.

13. Huber, D. L., Phys. Rev., 170, 418 (1968).

14. P euker, K., Trif on ov, E. D., Phys. stat. sol., 30, 479 (1968); Пойке р K. K., Трифонов Е. Д., ФТТ, 10,1705 (1968); Соколов И. В., Трифон о в Е. Д., Опт. и опектр., 30, 730 (1971).

15. T о у о $\mathrm{z}$ a w a, Y., J. Phys. Soc. Japan, 41, 400 (1976). 
16. Kubo, R., Takagawara, T., Hanamura, E., Oji Seminar on Physics of Highly Excited States in Solids, Hokkaido, Sept., 1975.

17. Hizhnyakov, V. V., Tehver, I. J., Preprint FI-31, Tartu, 1974; Tex в ер И. Ю., Хи жня ков В. В., ЖӘТФ, 69, 599 (1975).

18. Т ех в ер И. Ю., Препринт ИФ-5, Тарту, 1977.

19. В а в и лов С. И., Собр. соч., т. 2, М., 1952, с. 188.

20. Г а й т л е р В., Квантовая теория излучения, М., 1956, с. 192.

21. М а т у к Р., Фейнмановские диаграммы в проблеме многих тел, М., 1969.

22. Т ехвер И. Ю., Некоторые вопросы теории комбинационного рассеяния примесными центрами кристаллов, Канд. дис., Тарту, 1968.

23. Г лаубер Р., В сб.: Квантовая оптика и квантовая радиофизика, М., 1966, c. 91 .

24. Hizhnyakov, V. V., Tehver, I. J., In: Physics of Impurity Centres in Crystals, Tallinn, 1972, p. 607.

25. З а в Г. С., Теория колебаний кристаллической решетки, Тарту, 1966.

26. L a x, M., J. Chem. Phys., 20, 1752 (1952).

27. D exter, D., Phys. stat. sol. (b), 51, 571 (1972).

28. Dexter, D., Klick, C., Russell, G., Phys. Rev., 100, 603 (1955).

29. Р е б а н К. К., Тр. ИФА АН ЭССР, 7, 62 (1958).

30. К ролль Н., В сб.: Квантовая оптика и квантовая радиофизика, М., 1966, c. 66 .

Ннститут физики

Академии наук Эстонской ССР

Поступила в редакцию

17/XII 1976

\section{KRISTALLIDE LISANDITSENTRITE SEKUNDAARKIIRGUSE ULEMINEKUSPEKTRITE TEOORIA RESONANTSI KORRAL}

Esitatakse kristallide lisanditsentrite sekundaarkiirguse (SK) ajast sõltuvate üleminekuspektrite teooria resonantsi korral. Nimetatud spektrid kiiratakse lisanditsentri ergastatud elektronseisundis toimuva võnkerelaksatsiooni jooksul. Selgub, et suurte Stokesi kadude korral on SK silutud üleminekuspektritel normaaljaotuse kuju, kusjuures maksimumide asukoht ja spektrite laius muutuvad ajas. Ajalised sōltuvused määrab impulsi tüüpi foononite korrelatsioonfunktsioon ja neil on kustuvate võnkumiste iseloom, kusjuures võnkeperioodid kuuluvad pikosekundite ja subpikosekundite diapasooni. Esitatakse resonantsijuhulise SK silutud statsionaarse spektri lihtne valem, mis kirjeldab nii harilikku kui ka kuuma luminestsentsi. Teooriat on illustreeritud arvutustega.

\section{HIZHNYAKOV, Inna REBANE}

\section{THEORY OF TRANSIENT RESONANCE SECONDARY EMISSION SPECTRA OF IMPURITY CENTRES IN CRYSTALS}

A theory of time-dependent (transient) resonance secondary emission spectra (RSE) of impurity centres in crystals has been proposed. These spectra are emitted in the course of vibrational relaxation in the excited electronic state of the centre. It has been shown that, in the case of large Stokes' losses, the smoothed RSE spectra are described by a Gaussian curve whose place of the maximum and the width change with time. The corresponding time dependences are determined by an impulse-type phonon correlation function and are of the character of damping oscillations with periods in the picosecond and subpicosecond region. A simple formula has also been obtained for the smoothed stationary RSE spectrum containing ordinary as well as hot luminescence. The results of the theory are amply illustrated by calculations. 